\title{
Ectrópio congênito: relato de três casos e revisão de literatura
}

\author{
Congenital ectropion: three case reports and literature review
}

\author{
Filipe José Pereira ${ }^{1}$ \\ Sabrina de Pellegrini Trindade ${ }^{2}$ \\ Antonio Augusto Velasco e $\mathrm{Cruz}^{3}$
}

\begin{tabular}{|l|}
\hline RESUMO \\
\hline Relato de três casos de ectrópio congênito devido a sua raridade e confusa \\
classificação. Caso 1: JPT, 2 dias, masculino, negro. Apresentava eversão \\
de pálpebra superior esquerda com quemose, passível de redução mecâ- \\
nica. Resolução após 48 horas de oclusão compressiva. Caso 2: AJL, 6 \\
anos, feminino, branco, com síndrome de Down. Apresentava hiperemia, \\
lagoftalmo e leucoma inferior em olho esquerdo, sendo realizada cirurgia \\
de encurtamento horizontal ("tarsal strip" superior e inferior) complemen- \\
tada com enxerto de pele, sendo perdido o acompanhamento dois meses \\
após a cirurgia. Caso 3: GSD, 4 anos, masculino, branco, com síndrome de \\
Down. Apresentava mesmo quadro e tratado com mesma cirurgia bilateral. \\
Oprimeiro caso, conforme classificação dePicó,é grau II que ocorre devido \\
a eversão das pálpebras durante passagem pelo canal do nascimento, mais \\
freqüentemente encontrados em negros, e tratado quase exclusivamente \\
clinicamente. E os segundo e terceiro casos representam grau III, decor- \\
rente da alteração da pele palpebral, cuja associação à síndrome de Down \\
é bem estabelecida, tratado exclusivamente com cirurgias como as realiza- \\
das aqui. Discordamos da classificação de Picó, a única existente na \\
literatura, pois o grupo I (ausência de tarso) não possui nenhum artigo \\
científico comprovando sua existência, o grupo II deveria ser denominado \\
como eversão palpebral congênita, o grupo IV (microftalmia e cisto \\
orbitário) trata de doença primariamente orbitária. O grupo III refere-se ao \\
ectrópio verdadeiro, diagnosticado pelo alongamento horizontal das pál- \\
pebras superiores e inferiores (megalobléfaro). \\
\hline
\end{tabular}

Descritores: Ectrópio/congênito; Síndrome de Down/complicações; Pestanas/anormalidades; Pálpebras/cirurgia

\section{INTRODUÇÃ̃O}

A origem da palavra ectrópio é grega (ektrópion) e significa “girar para fora", referindo-se à eversão de qualquer parte do corpo, mas se consagrou o termo quando aplicado à margem palpebral ${ }^{(1)}$.

Quando presente ao nascimento é denominado ectrópio congênito, e poucos casos têm sido descritos desde 1896, data do primeiro relato de caso por Adams ${ }^{(2)}$. Em 1957, Picó propôs uma classificação de ectrópio congênito dividindo-o em 4 grupos de acordo com sua etiologia: I) devido à ausência ou anomalia do tarso; II) devido à eversão das pálpebras durante a passagem pelo canal do nascimento (edema palpebral causado por tocotraumatismo); III) devido à alteração da pele da pálpebra e IV) devido à microftalmia e ao cisto orbitário $^{(3)}$. O mesmo autor se refere aos grupos I e II como ectrópio verdadeiro e aos grupos III e IV como ectrópio secundário. Em 1964, Duke- 
Elder cita casos secundários a microftalmia, buftalmia e cistos, referindo-se aos casos primários como raridade ${ }^{(4)}$.

Por se tratar de uma doença rara, há muita controvérsia para que seja definido o que seria um ectrópio verdadeiro. Não há na literatura artigos que diferenciem ectrópio de uma eversão palpebral congênita, embora alguns enfatizem a diferença entre as duas alterações e a importância de não serem apontadas como sinônimos ${ }^{(5)}$. Assim como não há referências sobre alterações de volume orbitário como não sendo um quadro de ectrópio, também não há uniformidade na denominação de alterações encontradas ao exame, como megalobléfaro.

Conseqüentemente, sua patogênese ainda permanece obscura, apesar de já terem sido descritos fatores anatômicos e mecânicos associados ao ectrópio congênito, como o mecanismo de trauma facial com a parede uterina no momento do parto, ocasionando eversão das pálpebras e subseqüente edema resultante da obstrução do fluxo venoso palpebral ${ }^{(6-7)}$. Mas é possível que o evento inicial em todos os casos seja o espasmo palpebral, considerando que uma tensão excessiva da porção pré-septal do músculo orbicular levaria ao engurgitamento venoso com conseqüente edema conjuntival ${ }^{(8-9)}$. Em alguns casos de ectrópio congênito relatados na literatura é clara a descrição da piora da eversão palpebral em situação de choro exacerbado ou fechamento forçado das pálpebras, reforçando esta hipótese ${ }^{(8-10)}$.

Esta alteração palpebral já foi comprovadamente associada com afecções dermatológicas como a ictiose lamelar ${ }^{(11)} \mathrm{e}$ alterações cromossômicas como a síndrome de Down ${ }^{(9-10,12-13)}$.

Considerando o número pequeno de casos registrados na literatura, sua confusa classificação e sua desconhecida etiopatogenia, é proposto a seguir o relato e a discussão de três casos de ectrópio congênito.

\section{RELATO DOS CASOS}

\section{Caso 1}

J.P.T., 2 dias, masculino, negro, encaminhado pelo neonatologista por anormalidade palpebral. A criança havia nascido de parto normal, a termo, sem doenças associadas. Ao exame apresentava eversão de pálpebra superior esquerda com quemose de conjuntiva palpebral, sendo passível de redução mecânica, persistindo edema palpebral (Figura 1). Procedeu-se tampão oclusivo compressivo com regressão do ectrópio em 48 horas.

\section{Caso 2}

A.J.L., 6 anos, feminino, branca, procedente de Joaçaba (SC), encaminhada por alteração palpebral. A criança havia nascido de parto normal, a termo, com estigmas de síndrome de Down, não realizado o estudo citogenético. Ao exame oftalmológico apresentava hiperemia conjuntival bilateral, secreção mucóide e, apesar de lagoftalmo de cerca de $5 \mathrm{~mm}$, bom reflexo de Bell em ambos olhos que garantiu ausência de lesões corneanas ativas, mas com pequeno leucoma inferior em olho esquerdo. Foi realizada cirurgia de encurtamento ho- rizontal (através da técnica de tarsal strip superior e inferior após ressecção de cerca de $10 \mathrm{~mm}$ de espessura total de porção temporal de pálpebra esquerda) complementada com enxerto de pele (retirada da região retro-auricular ipsilateral) de pálpebras superior e inferior à esquerda, com bom resultado estético e funcional (Figura 2). Aos 2 meses de pós-operatório foi o último retorno da paciente, descontinuando o tratamento e faltou à cirurgia de pálpebras direita.

\section{Caso 3}

G.S.D., 4 anos, masculino, branco, procedente de Lages (SC), encaminhado por anormalidade palpebral. A criança ha-
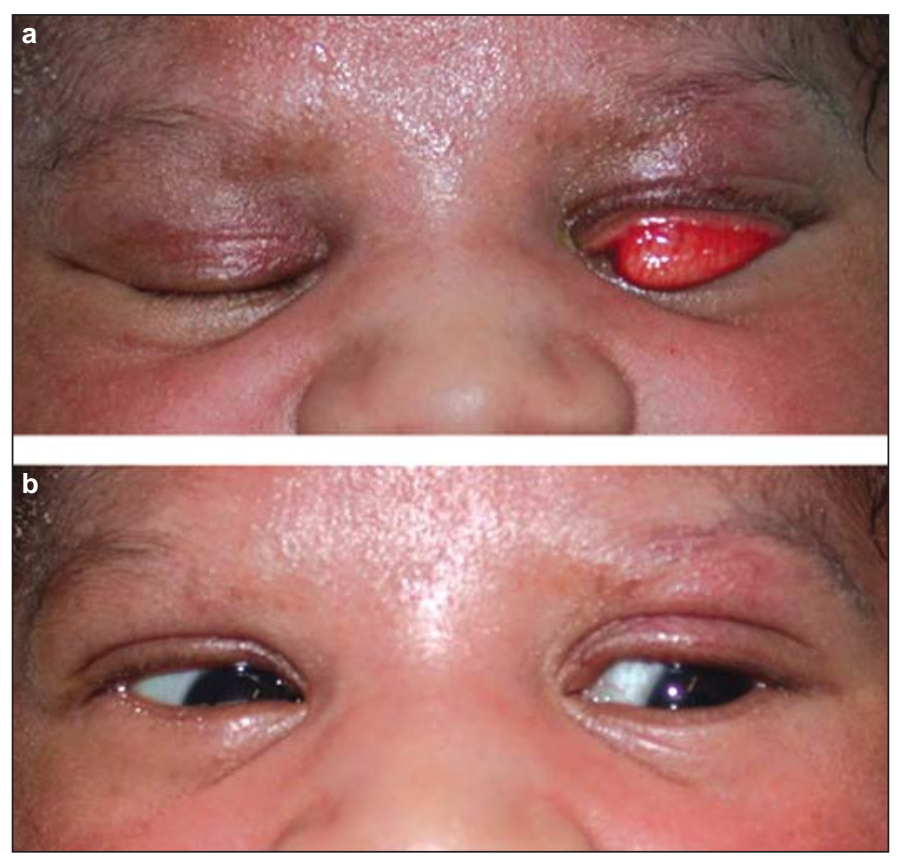

Figura 1 - a) eversão congênita de pálpebra superior esquerda; b) após regressão mecânica
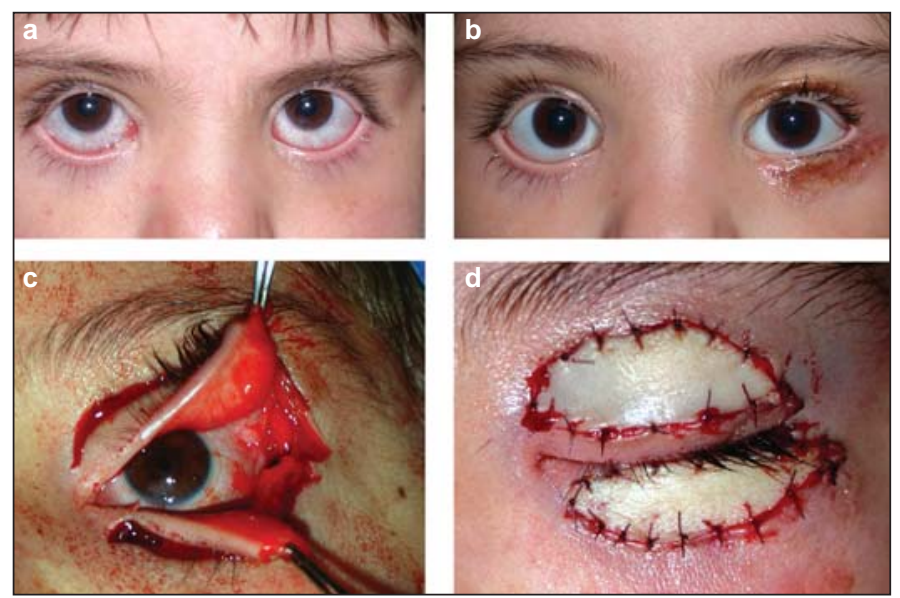

Figura 2 - a) pré-operatório; b) pós-operatório de dois meses de pálpebras esquerda; c) confecção de tarsal strip superior; d) sutura de enxertos em pálpebras superior e inferior 

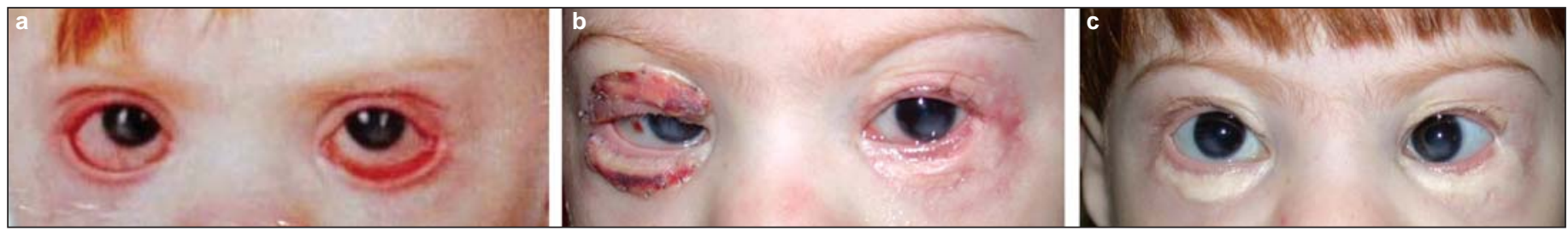

Figura 3 - a) pré-operatório; b) pós-operatório - 10 dias pálpebras direita e 2 meses e 10 dias de pálpebras esquerda ; c) pós-operatório - 6 meses após

via nascido de parto normal, a termo, com estigmas de síndrome de Down, confirmado ao estudo citogenético $47 \mathrm{XY}$, $+21 / 46$, XY; e após avaliação oftalmológica nas primeiras semanas foi diagnosticada úlcera corneana bilateral tratado com sucesso com colírio antibiótico ofloxacino a $0,3 \%$ (Oflox $\left.{ }^{\circledR}\right)$ e lubrificantes $\left(\right.$ Genteal $^{\circledR}$ e Lacrigel $\left.\mathrm{A}^{\circledR}\right)$. Foi encaminhado ao setor de oculoplástica, sendo realizado cirurgia de encurtamento horizontal (através da técnica de tarsal strip superior e inferior após ressecção de cerca de $10 \mathrm{~mm}$ de espessura total de porção temporal de pálpebras esquerda) complementada com enxerto de pele (retirada da região de fossa supra-clavicular ipsilateral) de pálpebras superior e inferior à esquerda; e 2 meses após, mesmo procedimento à direita, com bom resultado funcional e estético, persistindo ainda pequeno lagoftalmo noturno sem repercussões devido bom reflexo de Bell.

\section{DISCUSSÃO}

Descrevemos três casos de ectrópio congênito, sendo dois do sexo masculino e um do sexo feminino, todos nascidos de parto normal; a literatura disponível demonstra uma preferência pelo sexo masculino, já a natureza do parto não é considerada parte essencial do mecanismo de eversão palpebral se considerarmos alguns casos em que os bebês nasceram por parto cesáreo $^{(8,14)}$.

Aqui descrevemos um caso grau II (caso 1) e dois casos grau III (casos 2 e 3) de acordo com a classificação de Picó. Sua classificação é ainda muito confusa, e há muita controvérsia para que seja definido o que seria um ectrópio verdadeiro.

No primeiro caso é evidenciada a relação entre ectrópio grau II em criança negra, assim como a unilateralidade que não é a regra, mas é mais evidenciada em negros ${ }^{(7-8)}$. Bentsi-Enchill em sua amostra de 14 bebês negros observou que nos 6 casos de acometimento unilateral a pálpebra contralateral (sem eversão) apresentava uma sobreposição da pálpebra superior em relação a inferior, sendo proposto então que em negros os casos unilaterais poderiam ser explicados pela assimetria de sobreposição das pálpebras, levando-se em conta que a posição da face do feto no útero em conjunto com as contrações uterinas facilitariam a eversão palpebral ${ }^{(14)}$. Considerando a raridade desta condição palpebral e a significativa relação com a raça negra podese estabelecer uma etiologia ligada a fatores presdisponentes inerentes às pálpebras dos bebês acometidos ${ }^{(13-14)}$.

No segundo e terceiro casos observa-se a associação do ectrópio congênito com a síndrome de Down, já bem estabelecida, embora sem esclarecimento da etiopatogenia, inclusive em relatos no Brasil( ${ }^{(9-10,12-13)}$.

O tratamento do grau II é quase exclusivamente clínico, através de curativo oclusivo compressivo após redução mecânica do ectrópio, com o cuidado para a pálpebra não everter sob o curativo, ou simplesmente aguardar resolução espontânea, método mais demorado. Ocasionalmente é necessário correção cirúrgica provisória, como tarsorrafia. Em relação à manobra de redução mecânica do ectrópio deve-se estar atento à possibilidade de parada cardiorespiratória via reflexo oculorrespiratório, já descrita na literatura, sendo necessária a presença de profissional habilitado em ressucitação pediátrica durante o procedimento ${ }^{(7)}$. Em estudo comparativo do uso de tarsorrafia e injeção subconjuntival de hialuronidase seguida de tarsorrafia foi comprovado que na primeira situação a reversão das pálpebras se deu em cerca de 3 a 4 dias e com o tratamento combinado a reversão foi mais rápida, em torno de 1 a $2 \operatorname{dias}^{(14)}$. Assim como realizado neste trabalho, qualquer que seja o tratamento proposto, este deve ser precoce para diminuir o risco de ambliopia, especialmente em casos unilaterais $^{(7)}$. De forma semelhante outras alterações podem ser evitadas com a correção precoce da eversão palpebral, como blefarite, hiperceratose conjuntival, opacidade corneana que foram observadas em caso relatado de apresentação tardia de ectrópio congênito em criança de 11 anos de idade portador de síndrome de Down. Neste caso, em especial, ficou claro o papel exercido pelo músculo orbicular, agindo como esfíncter, perpetuando o ectrópio ${ }^{(13)}$.

O tratamento proposto para o grau III é sempre cirúrgico, como o realizado nesse trabalho, havendo variações quanto à técnica cirúrgica, estando de acordo com a apresentação clínica, sendo indispensável o encurtamento horizontal associado a correções como enxertos de pele, correção de ptose associados, entre outros ${ }^{(15)}$.

Portanto, discordamos da classificação de Picó, a única existente na literatura científica, pois o grupo I não possui nenhum artigo científico comprovando sua existência, o grupo II deveria ser denominado como eversão palpebral congênita, o grupo IV trata de uma patologia primariamente orbitária afetando secundariamente as pálpebras. O grupo III refere-se ao ectrópio verdadeiro e teria como característica associada em todos os casos relatados o alongamento horizontal das pálpebras superiores e inferiores, denominado megalobléfaro. 


\section{ABSTRACT}

To report 3 cases of congenital ectropion because of their rarity and confusing classification. Case 1: JPT, 2 days old, male, negro. Left upper eyelid eversion with chemosis was present, passive to mechanic reduction. Compressive occlusion was done with ectropion regression in 48 hours. Case 2: AJL, 6 years old, female, Caucasian, with Down syndrome. The left eye had hiperemia, lagophthalmos and inferior leucoma. She received horizontal shortening (superior and inferior tarsal strip) and skin grafts, and after 2 months the patient did not return. Case 3: GSD, 4 years old, male, Caucasian, with Down syndrome. His signs and treatment were the same as in case 2. According to Picó's classification the first case is classified as grade II due to eyelid eversion during the passage through the birth canal, more frequent in black people. Cases 2 and 3 represent grade III that is due to eyelid skin alteration, and the association with Down syndrome is observed. Treatment for ectropion grade III is always surgical, as it was done in these cases. We do not agree with Picó's classification, the only one found in medline, because there are no articles confirming the existence of grade I (absent tarsus), grade II should be called congenital upper eyelid eversion, grade IV (microphthalmos and orbital cyst) is a disease of the orbit. Grade III refers to true ectropion, because horizontal enlargement of superior and inferior eyelids (megaloblepharon).

Keywords: Ectropion/congenital; Down's syndrome/complications; Eyelashes/abnormalities; Eyelids/surgery

\section{REFERÊNCIAS}

1. França VP. Ectrópio. In: Soares EJC, Cirurgia plástica ocular. São Paulo: Roca; 1997. p.156-9.

2. Adams AL. A case of double congenital ectropion. Med Fortnightly. 1896; 9:337-8.

3. Pico G. Congenital ectropion and distichiasis; etiologic and hereditary factors: a report of cases and review of the literature. Trans Am Ophthalmol Soc. 1957-1958;55:663-700.

4. Duke-Elder S. Congenital anomalies of the ocular adnexa: anomalies of the eyelids. In: Duke-Elder S. System of Ophthalmology. Normal and abnormal development. London: Henry Kimpton; 1964. v.3 p.841.

5. Gershanik JJ, Baucum RW Jr. Primary congenital eversion of the eyelids. Am J Dis Child. 1974;127(2):243-4.

6. Morax S, Gatinel D. [Primary congenital ectropion. A propos of 2 cases] J Fr Ophtalmol. 1996;19(6-7):415-22. French.

7. Watts MT, Dapling RB. Congenital eversion of the upper eyelid: a case report. Ophthal Plast Reconstr Surg. 1995;11(4):293-5.

8. Raab EL, Saphir RL. Congenital eyelid eversion with orbicularis spasm. J Pediatr Ophthalmol Strabismus. 1985;22(4):125-8.

9. Johnson CC, McGowan BL. Persistent congenital ectropion of all four eyelids with megaloblepharon. Am J Ophthalmol. 1969;67(2);252-6.

10. Gilbert HD, Smith RE, Barlow MH, Mohr D. Congenital upper eyelid eversion and Down's syndrome. Am J Ophthalmol. 1973;75(3):469-72.

11. Jordan DR, Bawazeer AM, Pelletier CR. Severe congenital ectropion secondary to lamellar ichthyosis. Can J Ophthalmol. 1998;33(1):30-1.

12. Brik D, Cenovicz MT, Cruz GAO. Euribléfaro e ectrópio congênitos associados a síndrome de Down. Arq Bras Oftalmol. 1990;55(5):219-21.

13. Sellar PW, Bryars JH, Archer DB. Late presentation of congenital ectropion of the eyelids in a child with Down syndrome: a case report and review of the literature. J Pediatr Ophthalmol Strabismus. 1992;29(1)64-7.

14. Bentsi-Enchill KO. Congenital total eversion of the upper eyelids. Br J Ophthalmol. 1981;65(3):209-13.

15. Pelletier CR, Jordan DR, Konzuk PJ, Pashby RC. Tarsal eversion: a dramatic form of ectropion at birth. Can J Ophthalmol. 1996;31(6):313-4.

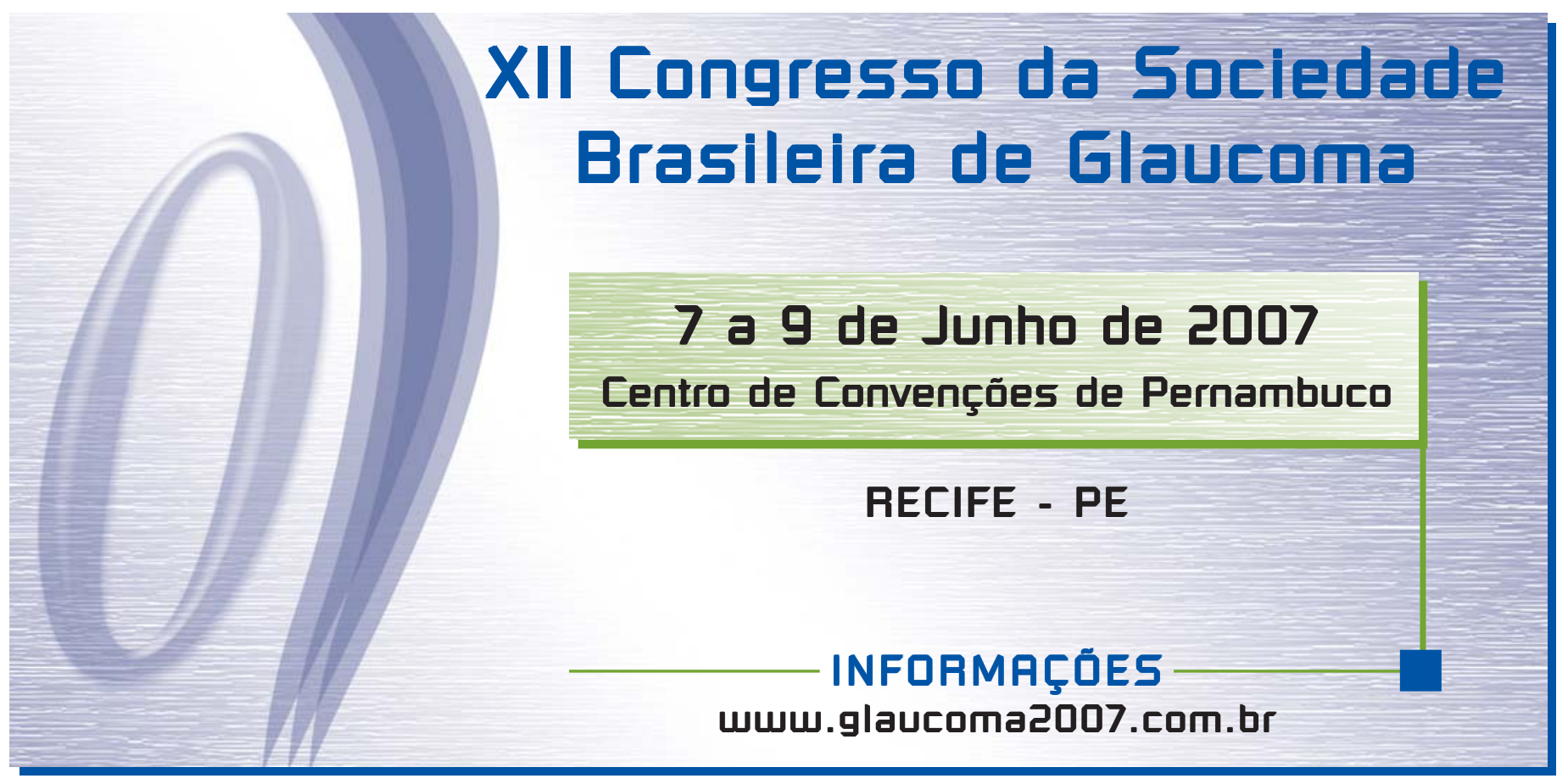

